\title{
COMPUTATIONS FOR A VIBRATING SYSTEM DIAGONALIZE THE VARIANCE
}

\author{
J. N. BOYD and P. N. RAYCHOWDHURY \\ Department of Mathematical Sciences \\ Virginia Commonwealth University \\ Richmond Virginia 23284-2014 \\ (Received August 11, 1993 and in revised form September 15, 1993)
}

ABSTRACT. The transformations to diagonalize potential energy matrices for coupled harmonic oscillators will also diagonalize the variance when written in matrix form. After a brief review of a geometrical interpretation of the variance, the transformations are described and an example is given.

KEY WORDS AND PHRASES. Variance, orthogonal and unitary transformations, coupled oscillators, elastic potential energy.

1991 AMS Subject Classification Code. Primary 20C99, Secondary $20 \mathrm{C} 35$.

INTRODUCTION. In this note, we will be interested in $\mathrm{n}$ independently chosen real numbers $x_{1}, x_{2}, \ldots, x_{n}$ with mean $\bar{x}=\frac{1}{n} \sum_{i=1}^{n} x_{i}$ and in their variance

$$
s^{2}=\frac{1}{n-1} \sum_{i=1}^{n}\left(x_{i}-\bar{x}\right)^{2}
$$

which can also be written as

$$
s^{2}=\frac{1}{n-1}\left(\sum_{i=1}^{n}\left(x_{i}\right)^{2}-\frac{1}{n}\left(\sum_{i=1}^{n} x_{i}\right)^{2}\right)
$$

We begin by recalling a geometric interpretation for $\mathrm{s}^{2}$.

Let the n-tuple $\left(x_{1}, x_{2}, \ldots, x_{n}\right)$ represent a point $P$ in $n$-dimensional Euclidean space $(E(n))$ and let orthogonal unit vectors $\hat{u}_{1}, \hat{u}_{2}, \ldots, \hat{u}_{n}$ be defined in the positive $x_{1}, x_{2}, \ldots, x_{n}$ directions, respectively. Then the vector $\vec{v}=x_{1} \hat{u}_{1}+x_{2} \hat{u}_{2}+\ldots+x_{n} \hat{u}_{n}$ can be taken to emanate from the origin $\mathrm{O}:(0,0, \ldots, 0)$ and to terminate at $P$.

The projection of $\vec{v}$ upon the line $\ell: x_{1}=x_{2}=\ldots=x_{n}$ is given by the inner product $\vec{v} \cdot\left(\frac{\hat{u}_{1}+\hat{u}_{2}+\ldots+\hat{u}_{n}}{\sqrt{n}}\right)=\frac{1}{\sqrt{n}} \sum_{i=1}^{n} x_{i}$ as suggested in Figure 1. In n-dimensions, the distance (d) from $P$ to $\ell$ satisfies the equation

$$
d^{2}=\sum_{i=1}^{n}\left(x_{i}\right)^{2}-\frac{1}{n}\left(\sum_{i=1}^{n} x_{i}\right)^{2}=(n-1) s^{2} .
$$

Thus $\mathrm{s}^{2}$ is simply proportional to the square of the distance from $P$ to $\ell$. To pursue the simplification, we first rewrite Equation 1 in matrix form: 


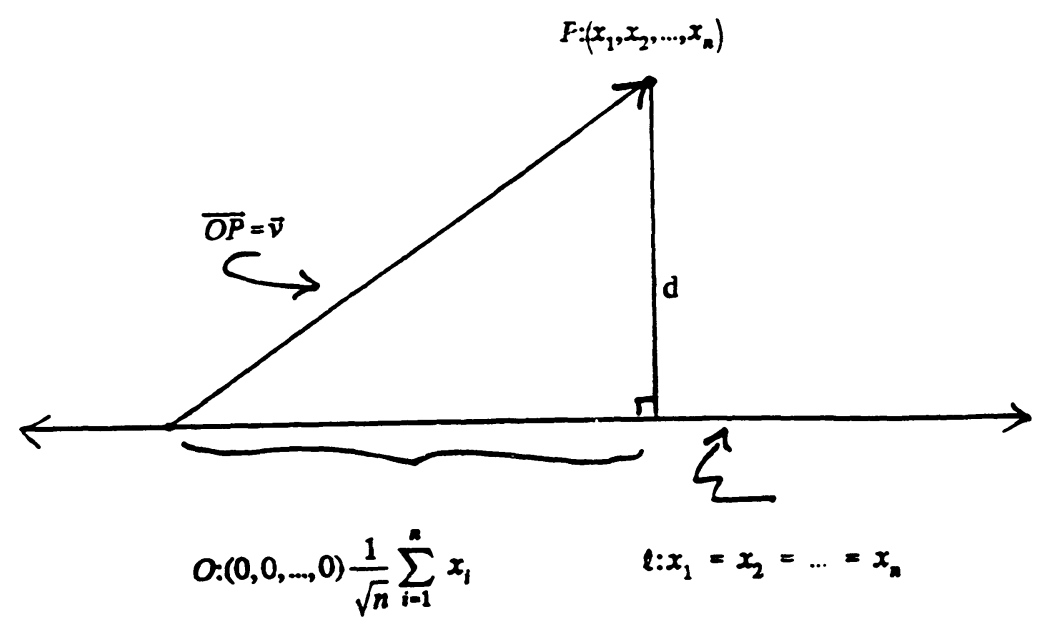

Figure 1: The Geometry of $P, \vec{\nabla}$, and $\ell$.

$$
(n-1) s^{2}=\frac{1}{n}\left(x_{1} x_{2} x_{3} \cdots x_{n}\right)\left(\begin{array}{ccccc}
n-1 & -1 & -1 & \cdots & -1 \\
-1 & n-1 & -1 & \cdots & -1 \\
-1 & -1 & n-1 & \cdots & -1 \\
\vdots & \vdots & \vdots & & \vdots \\
-1 & -1 & -1 & \cdots & n-1
\end{array}\right)\left(\begin{array}{c}
x_{1} \\
x_{2} \\
x_{3} \\
\vdots \\
x_{n}
\end{array}\right)
$$

Our problem is to diagonalize the matrix.

AN EXAMPLE. To illustrate the calculations, let us consider the case for $n=4$. Then

$$
f\left(x_{1}, x_{2}, x_{3}, x_{4}\right)=3 s^{2}=\frac{1}{4}\left(x_{1} x_{2} x_{3} x_{4}\right)\left(\begin{array}{rrrr}
3 & -1 & -1 & -1 \\
-1 & 3 & -1 & -1 \\
-1 & -1 & 3 & -1 \\
-1 & -1 & -1 & 3
\end{array}\right)\left(\begin{array}{l}
x_{1} \\
x_{2} \\
x_{3} \\
x_{4}
\end{array}\right)
$$

In $\mathrm{E}(4)$, we imagine surfaces of constant $f\left(x_{1}, x_{2}, x_{3}, x_{4}\right)$ to be concentric cylinders with axis $\ell$.

It is clear that in choosing new coordinates, we must take one coordinate to be proportional to $x_{1}+x_{2}+x_{3}+x_{4}$ to place one axis on $\ell$. Simple choices of the other three are proportional to $x_{1}-x_{2}, x_{3}-x_{4}$ and $-x_{1}-x_{2}+x_{3}+x_{4}$ to determine the orthogonal transformation matrix

$$
S=\left(\begin{array}{cccc}
\frac{1}{\sqrt{2}} & -\frac{1}{\sqrt{2}} & 0 & 0 \\
0 & 0 & \frac{1}{\sqrt{2}} & -\frac{1}{\sqrt{2}} \\
\frac{1}{2} & \frac{1}{2} & -\frac{1}{2} & -\frac{1}{2} \\
\frac{1}{2} & \frac{1}{2} & \frac{1}{2} & \frac{1}{2}
\end{array}\right)
$$


We can rewrite Equation 4 as

$$
\begin{aligned}
& \frac{1}{4}\left(x_{1} x_{2} x_{3} x_{4}\right) S^{-1} S\left(\begin{array}{rrrr}
3 & -1 & -1 & -1 \\
-1 & 3 & -1 & -1 \\
-1 & -1 & 3 & -1 \\
-1 & -1 & -1 & 3
\end{array}\right) S^{-1} S\left(\begin{array}{l}
x_{1} \\
x_{2} \\
x_{3} \\
x_{4}
\end{array}\right)= \\
& \left(x_{1}^{\prime} x_{2}^{\prime} x_{3}^{\prime} x_{4}^{\prime}\right)\left(\begin{array}{llll}
1 & 0 & 0 & 0 \\
0 & 1 & 0 & 0 \\
0 & 0 & 1 & 0 \\
0 & 0 & 0 & 0
\end{array}\right)\left(\begin{array}{c}
x_{1}^{\prime} \\
x_{2}^{\prime} \\
x_{3}^{\prime} \\
x_{4}^{\prime}
\end{array}\right)=\left(x_{1}^{\prime}\right)^{2}+\left(x_{2}^{\prime}\right)^{2}+\left(x_{3}^{\prime}\right)^{2}
\end{aligned}
$$

where the new coordinates $x_{1}^{\prime}, x_{2}^{\prime}, x_{3}^{\prime}$ combine to yield the square of the distance from $\ell$ which is now the $x_{4}^{\prime}$ axis. The appearance of only three summands is consistent with the $4-1=3$ degrees of freedom for the variance.

ANOTHER APPROACH. Let us now return to Equation 1. After a bit of algebra, we see that the equation for the variance can be rewritten yet again as

$$
s^{2}=\frac{1}{n-1} \cdot \frac{1}{n} \sum_{i=1}^{n-1} \sum_{j=i+1}^{n}\left(x_{i}-x_{j}\right)^{2}
$$

Having obtained Equation 5, we recognized it to be essentially the same as that for the elastic potential energy for $\mathbf{n}$ masses symmetrically arranged on a fixed circle and interconnected by idealized harmonic springs between all pairs of masses. Furthermore, we have previously described this system.[1]

Returning to the case $n=4$, let us consider the four masses on the circle as shown in Figure 2.

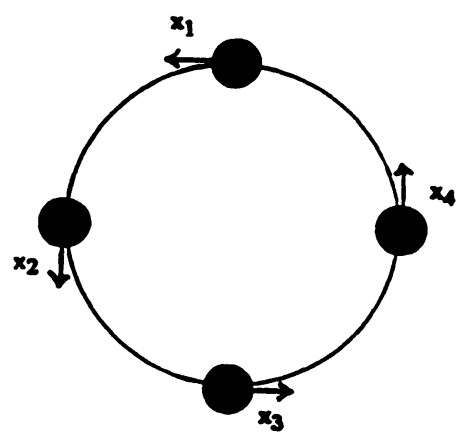

Figure 2. The Coupled Oscillators.

The particles are constrained to move on the circle and their displacements from 
their equilibrium positions are taken to be $x_{1}, x_{2}, x_{3}, x_{4}$. We choose the elastic constants for all couplings to be $k$. Then the potential energy can be written as

$$
\text { P.E. }=\frac{1}{2} k\left[\left(x_{1}-x_{2}\right)^{2}+\left(x_{1}-x_{3}\right)^{2}+\left(x_{1}-x_{4}\right)^{2}+\left(x_{2}-x_{3}\right)^{2}+\left(x_{2}-x_{4}\right)^{2}+\left(x_{3}-x_{4}\right)^{2}\right] \text {. }
$$

The symmetries which simplified the potential energy function were derived from the rotations of the circle. The same symmetries can be applied to $f\left(x_{1}, x_{2}, x_{3}, x_{4}\right)$ of Equation 4. The four-by-four matrix

$$
\left(\begin{array}{rrrr}
3 & -1 & -1 & -1 \\
-1 & 3 & -1 & -1 \\
-1 & -1 & 3 & -1 \\
-1 & -1 & -1 & 3
\end{array}\right)
$$

can be diagonalized by the unitary transformation having matrix representation

$$
U=\frac{1}{2}\left(\begin{array}{rrrr}
i & -1 & -i & 1 \\
-1 & 1 & -1 & 1 \\
-i & -1 & i & 1 \\
1 & 1 & 1 & 1
\end{array}\right)
$$

which was computed from the irreducible representations of the group of rotations by $90^{\circ}$ $180^{\circ}, 270^{\circ}$, and $360^{\circ}$ about the center of the circle of Figure 2. [2,3]

Although the transformation carries the coordinates into complex numbers, $f$ which is proportional to the variance remains fixed and real:

$$
\begin{aligned}
f\left(x_{1}, x_{2}, x_{3}, x_{4}\right) & =\frac{1}{4}\left(x_{1} x_{2} x_{3} x_{4}\right) U^{-1} U\left(\begin{array}{rrrr}
3 & -1 & -1 & -1 \\
-1 & 3 & -1 & -1 \\
-1 & -1 & 3 & -1 \\
-1 & -1 & -1 & 3
\end{array}\right) U^{-1} U\left(\begin{array}{l}
x_{1} \\
x_{2} \\
x_{3} \\
x_{4}
\end{array}\right) \\
& =\frac{1}{4}\left(y_{1} y_{2} y_{3} y_{4}\right)\left(\begin{array}{llll}
4 & 0 & 0 & 0 \\
0 & 4 & 0 & 0 \\
0 & 0 & 4 & 0 \\
0 & 0 & 0 & 0
\end{array}\right)\left(\begin{array}{c}
\overline{y_{1}} \\
\overline{y_{2}} \\
\overline{y_{3}} \\
\overline{y_{4}}
\end{array}\right) \\
& \left.=y_{1} \overline{y_{1}}+y_{2} \overline{y_{2}}+y_{3} \overline{y_{3}}\right)=r_{1}^{2}+r_{2}^{2}+r_{3}^{2}
\end{aligned}
$$

where $y_{t}, \bar{y}_{i}$, and $r_{i}$ represent the new normal coordinates, their complex conjugates, and their moduli, respectively. Thus the variance has again been diagonalized. In addition, we now have unitary matrices to diagonalize the variance matrix for all $n \geq 2$. They have already been computed to diagonalize potential energies.

GENERALIZATION. The purpose of this note is to point out that the unitary transformations developed from the group of rotations of the circle enable us to look at variance and 
standard deviation. We had previously used these transformations to find natural frequencies for oscillating mass and spring systems. We were pleased that previous work solved a new problem for us. We simply state the generalization. The variance for $n \geq 2$ real numbers $x_{1}, x_{2}, \cdots, x_{n}$ can be computed as

$$
\begin{aligned}
s^{2} & =\frac{1}{n-1}\left(\sum_{i=1}^{n}\left(x_{i}\right)^{2}-\frac{1}{n}\left(\sum_{i=1}^{n} x_{1}\right)^{2}\right) \\
& =\frac{1}{n-1} \cdot \frac{1}{n} \sum_{i=1}^{n-1} \sum_{j=i+1}^{n}\left(x_{i}-x_{j}\right)^{2} \\
& =\frac{1}{n-1} \cdot \frac{1}{n}\left(x_{1} x_{2} \cdots x_{n}\right)\left(\begin{array}{ccccc}
n-1 & -1 & -1 & \cdots & -1 \\
-1 & n-1 & -1 & \cdots & -1 \\
\vdots & \vdots & \vdots & & \vdots \\
-1 & -1 & -1 & \cdots & n-1
\end{array}\right)\left(\begin{array}{c}
x_{1} \\
x_{2} \\
\vdots \\
x_{n}
\end{array}\right) .
\end{aligned}
$$

The unitary matrix

$$
U=\frac{1}{\sqrt{n}}\left(\begin{array}{cccccc}
\exp \frac{2 \pi i}{n} & \exp \frac{4 \pi i}{n} & \exp \frac{6 \pi i}{n} & \ldots & \exp \frac{2(n-1) \pi i}{n} & 1 \\
\exp \frac{4 \pi i}{n} & \exp \frac{8 \pi i}{n} & \exp \frac{12 \pi i}{n} & \ldots & \exp \frac{4(n-1) \pi i}{n} & 1 \\
\vdots & \vdots & \vdots & & \vdots & \vdots \\
1 & 1 & 1 & \ldots & 1 & 1
\end{array}\right)
$$

which was developed from the representations of the Abelian group of rotations through $\frac{2 \pi}{n}$ of the circle in order to diagonalize a potential energy matrix will also diagonalize the variance matrix.

\section{REFERENCES}

1. BOYD, J. N. and RAYCHOWDHURY, P.N., A Group Theoretic Approach to Generalized Harmonic Vibrations in a One Dimensional Lattice, Int. J. of Math. and Math. Sci. 9 (1986), 131-136.

2. BOYD, J. N. and RAYCHOWDHURY, P.N., An Application of Projection Operators to a One Dimensional Crystal, Bull. of the Inst. of Math. Academia Sinica 7 (1979), 133-144.

3. BOYD, J. N. and RAYCHOWDHURY, P.N., Group Representations in Lagrangian Mechanics, Physica 114A (1982), 604-608. 


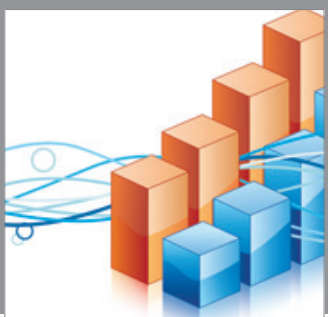

Advances in

Operations Research

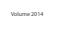

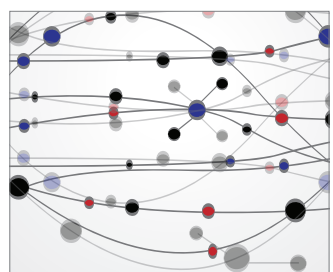

\section{The Scientific} World Journal
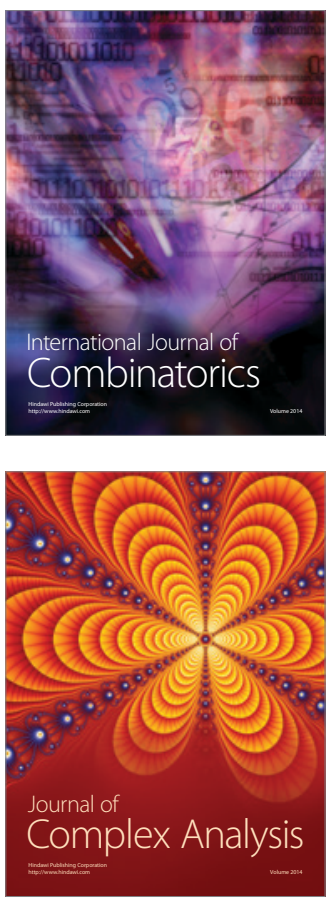

International Journal of

Mathematics and

Mathematical

Sciences
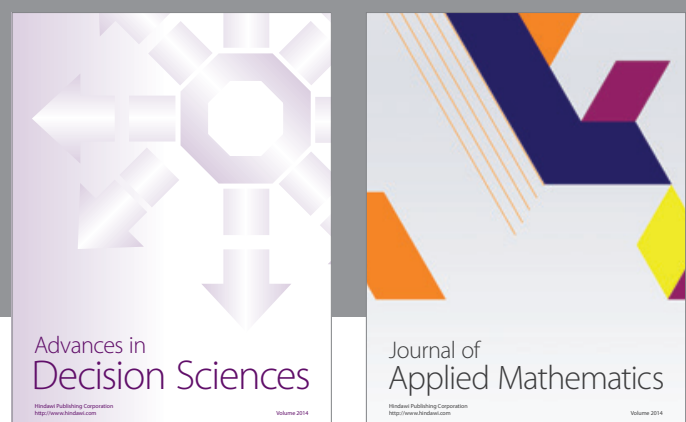

Journal of

Applied Mathematics
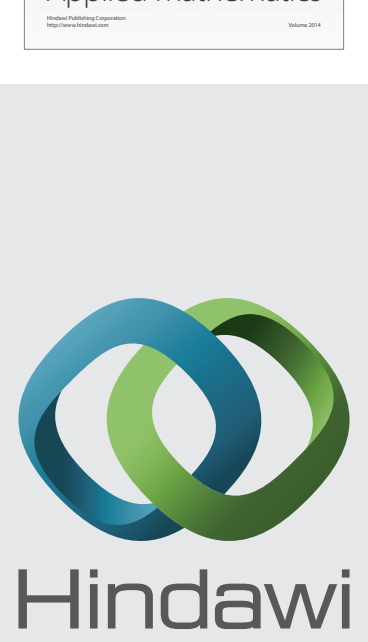

Submit your manuscripts at http://www.hindawi.com
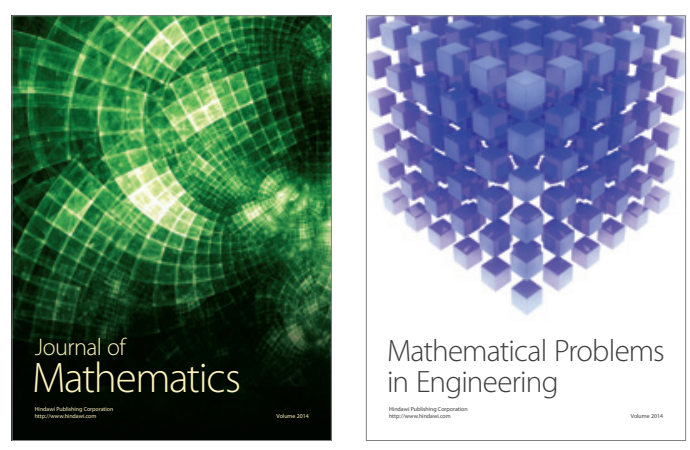

Mathematical Problems in Engineering
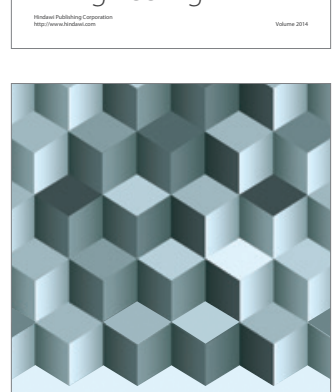

Journal of

Function Spaces
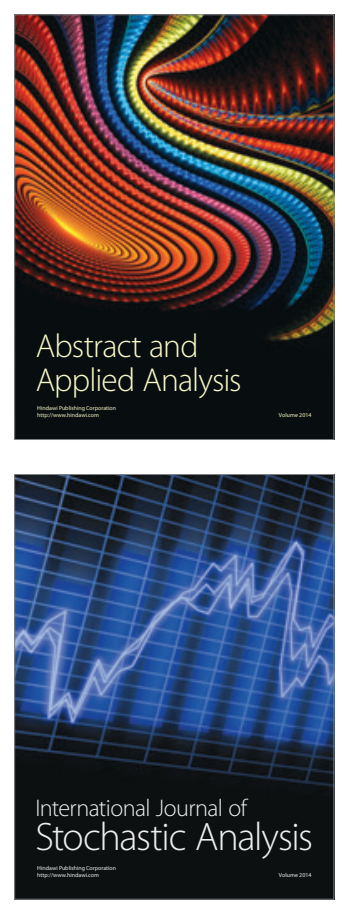

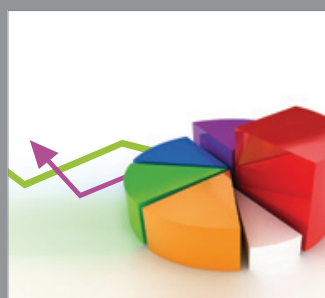

ournal of

Probability and Statistics

Promensencen
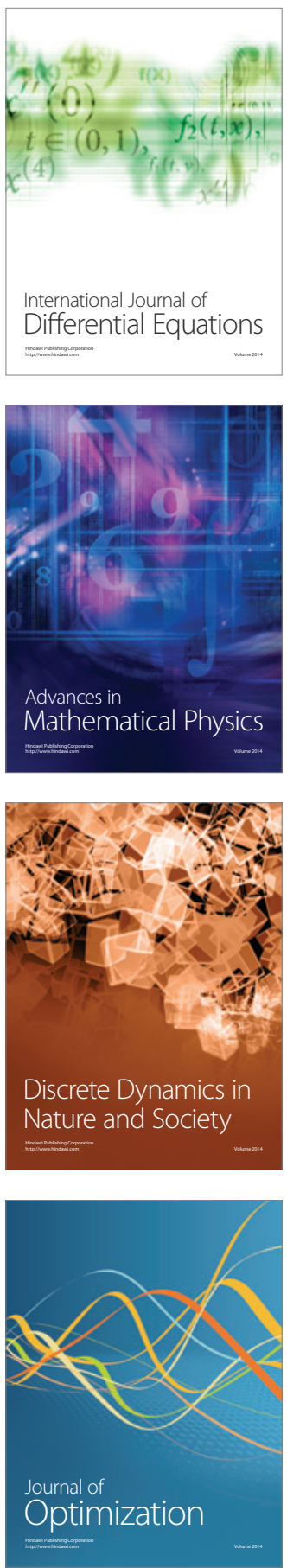